\title{
Application Analysis on Modern Machinery Manufacturing Technology and Precision Machining Technology
}

\author{
Xu Chengyi \\ Jiangxi Modern Polytechnic College, Nanchang, Jiangxi, 330056, China
}

Keywords: machinery manufacturing technology; precision machining technology; application analysis

\begin{abstract}
With the development of China's economy and progress of scientific and technological level, relevant industries of machinery manufacturing and precision machining is increasingly prosperous. Meanwhile, all sectors of the society put forward higher requirements for production technology and the quality of products. Traditional machinery manufacturing technology and machining technology cannot satisfy actual demands of modern society. Therefore, relevant enterprises shall combine with their own characteristics, continuously conduct technological innovations, introduce hi-tech means, enhance their own comprehensive competitive strength, improve the quality of products and realize intellectualized and automatic production process, so as to better serve economic construction.

With the deepening of market economic system, the market competition faced by machinery manufacturing enterprises is fiercer. In order to comprehensively enhance their core competitiveness and better adapt to changes of the situation, relevant enterprises are required to incessantly enhance the quality of products and production efficiency, improve work environment, promote safety performance of production, eliminate outdated technology and equipment, apply advanced equipment and technology for production, increase the application of modern machinery manufacturing technology and precision machining technology and realize their comprehensive development.
\end{abstract}

\section{The Significance of Modern Machinery Manufacturing Technology and Precision Machining Technology}

In general, modern machinery manufacturing technology includes technology to produce mechanical equipment and to process materials by cutting. Compared with traditional manufacturing technology, modern machinery manufacturing technology has characteristics of automation, digitization, informatization and high intellectualization, and can achieve the integration of maintenance, design and troubleshooting. Precision machining technology is full with high scientific and technological content, widely applied in high technology and industry. Mechanical design generally include material design, structure design, technological design and so on. Traditional design concept and production technology cannot satisfy the requirements of modern machinery design and production, such as steam turbine blade and numerical control machine. The design of modern machinery manufacturing has developed to be modern design from intuitive and experience design to solve different problems in actual design based on scientific methods and theoretical knowledge. Modern mechanical design includes design scheme optimization, reliability design, simulation, computer-aided design, systems engineering and other wide fields, can comprehensively improve work efficiency and overall design performance and promote the quality of products, and accelerates the development of related industries [1].

Modern machinery manufacturing and precision machining are widely applied in various fields, such as metallurgy, electronics, and mechanical engineering, with high attention and investment from countries in the world on the development of relevant technologies. The progress of related technologies is able to greatly promote industrial development and scientific and technological level, realize the application of virtual products to real life and improve people's living standard. At the 
same time, machinery manufacturing and precision machining belong to the most important element in production, and different technology will affect the overall quality of products, therefore, it is necessary to determine machine and production technology according to actual requirements of products, formulate reasonable goals, realize the improvement of enterprises' competitiveness and better adapt to changes of economic situation.

\section{Characteristics of Modern Machinery Manufacturing and Precision Machining}

Modern machinery manufacturing and precision machining technology are the outcomes of scientific and technological development, with certain systematicness. It is required to apply production automation technology, computer information technology, sensor technology and etc. in actual application of related technologies. And it is necessary to combine with new materials and technology and novel management method and integrate different technologies into one system to accomplish the whole production.

In addition to the manufacturing of products, the technology is advanced in the development, production, sales, design and after-sales of products. Different contents are related to each other. If there is problems in one link, other links will be affected, so there is a strong relevance. With the development of economic and trade globalization and the increasing degree of China's opening to the outside world, enterprises shall introduce international advanced technologies, improve their own performance and realize their comprehensive development [2].

\section{The Application of Modern Machinery Manufacturing}

\subsection{Submerged-arc welding}

Submerged-arc welding technology is to perform arc burning below flux layer for a period of time and implement welding, generally including semi-automatic and automatic welding. Semi-automatic welding requires welding wire to be manually put into place, with a certain input of manpower and relatively complicated operation process, so it is less applied by enterprises than automatic welding. In operation, it is necessary to strictly control the quality of flux, select reasonable type, and ensure the alkalinity of materials to meet the demand of production [3]. Compared with other welding technologies, the overall quality of submerged-arc welding is relatively high, without any smoke or arc light in operation, so it is widely applied in the welding of important structures, such as box beam column, tabulation, pressure vessel and so on. Specific principle is shown in Figure 1.

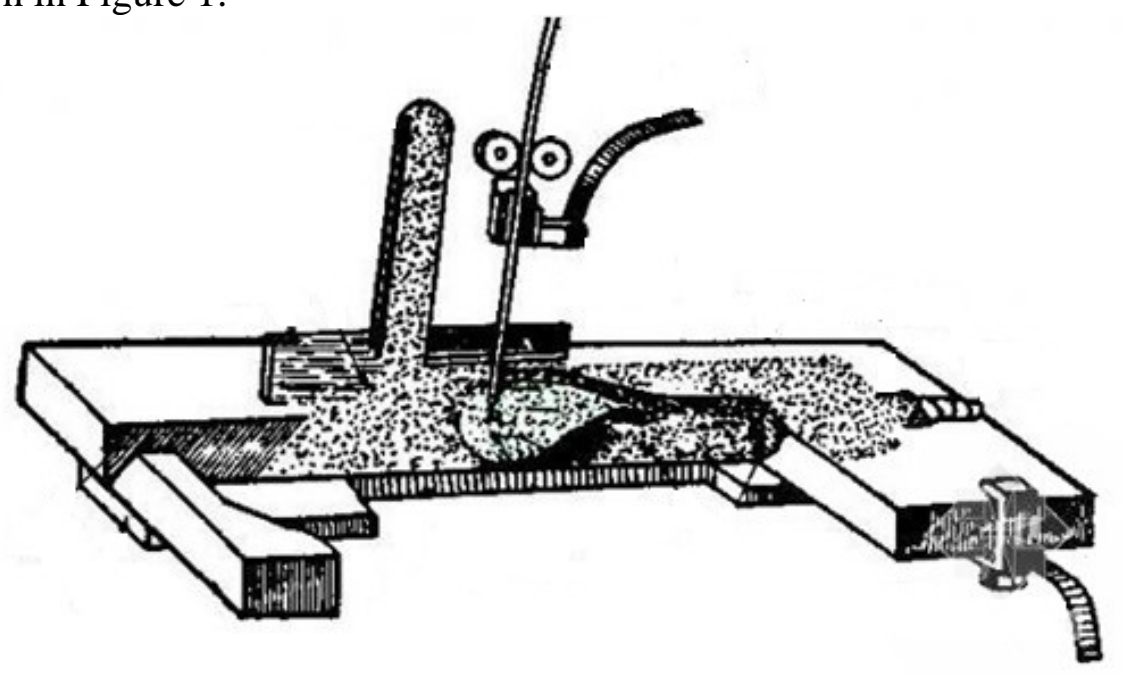

Figure 1 Schematic Diagram of Submerged-arc Welding

\subsection{Gas shielded welding}

Gas shielded welding, also called gas shielded arc welding, is an important type of current arc 
welding technology. Its main feature is to take gas as the medium to realize the better protection of arc and welding position and improve the quality of welding. At present, the gas medium most widely applied in actual production is carbon dioxide, which can effectively isolate the air and reduce the contact of air and welding position. This welding technology costs less resources, with low production cost, simple operation procedures, less slag and high welding efficiency, and can realize the intellectualization and automation of welding with less radiation quantity, and reduce the harm on the body of welding operation staff. However, the technology has relatively high operation requirements with high costs of related equipment.

\subsection{Electric resistance welding}

Basic operation principle of electric resistance welding is to perform compaction treatment on two workpieces to be welded between two electric machines. When power is connected, welding current will be generated between electrodes. When current goes through nearby area of workpiece and contact surface, there will be heating in resistance. If the heat reaches the molten or plastic state of resistance, metal ion on the surface will form metal chemical bond, form plenty of grain in compaction position and form welding seam or welding spot in joints. The main advantages are simple and flexible operation, less procedures, less time for heating, low production costs and low investment. However, constrained by current objective technological conditions, there is a lack of harmless and strongly reliable inspection method for this welding technology, and the maintenance of equipment is difficult [4].

\subsection{Friction stir welding and stud welding}

Friction stir welding belongs to a mature welding technology with a long history of application in airplane and automobile manufacturing and so on. This welding technology consumes less materials like welding rod and wire, realizes the requirements of energy conservation and environment protection, completes longer welding length when welding aluminum alloy, and requires lower welding temperature and simple operation, relatively widely applied in traditional and some new manufacturing industries.

Stud welding is mainly to make use of basic principle of pressure, increase the pressure of stud, connect power, melt object to be welded and realize final welding effect. It is divided into energy store type and draw arc type, the former is extensively used in thin plate welding and the latter is in heavy industry.

\section{The Application of Precision Machining Technology}

Precision machining technology can satisfy basic demand of people for the precision of mechanical products, mainly including precision cutting, micro machining, ultra precision grinding, nano-technology and so on.

\subsection{Precision cutting}

Precision cutting, a common precision machining technology, directly performs cutting on materials to be processed with certain equipment and realizes the requirements of products in thickness, width, length and other sizes, extensively applied in manufacturing industry. For instance, in order to realize the roughness requirements of product surfaces, it is required to control harmful impact of various factors from the outside on product surfaces, and to increase the machining precision so as to ensure the stiffness of machine tool equipment to meet the demand of production, without deformation in special conditions like high temperature, and with a certain aseismic capacity. In actual production, it is necessary to increase the rotational speed of spindle and continuously improve overall level of processing with precision control technology and precision positioning technology.

\subsection{Ultra precision grinding}

Ultra precision grinding is applied to meet the requirement of product with the surface roughness 
of $1-2 \mathrm{~mm}$ in production. It is commonly combined with silicon chip grinded and polished in the same level of atom to satisfy the demand of machining precision [6]. With the improving requirement for the product quality in all sectors, relevant enterprises shall innovate technologies in production, enhance the precision of technician and meet actual demand of social production. Figure 2 is schematic diagram of ultra precision grinding with magnetic grinding.

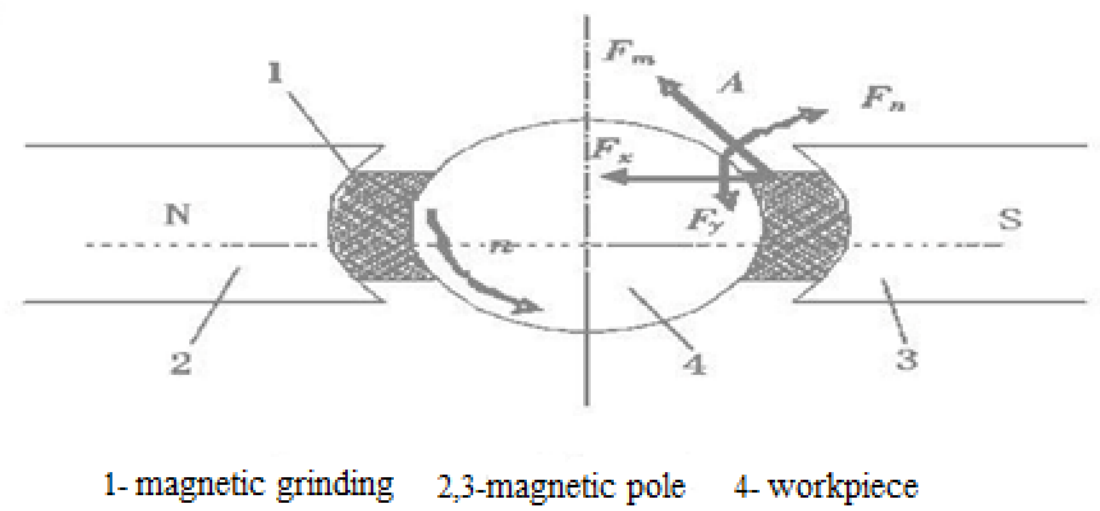

Figure 2 Schematic Diagram of Ultra Precision Grinding with Magnetic Grinding

\subsection{Micro machining and abrasive tool moulding}

With the development of information Internet and communication technology, various electronic mechanical equipment are increasingly used in actual production. With simple and light volume and small size, they consume less resources, which can achieve higher operational speed and precision and accurately complete all kinds of practical operations due to the development of micro machining technology. At present, it is widely used in the production of small electronic equipment. For instance, in the production of semi-conductor, micro machining technology is able to improve the quality and performance of products and help semi-conductor industry to achieve comprehensive progress and development.

Abrasive tool moulding technology also belongs to precision machining with an extensive application. A large portion of parts currently used in electronic products are processed by abrasive tools, which can enhance the precision of abrasive tools and further improve the quality of electronic products [7].

\subsection{Nano-technology}

Nano-technology, spatial information technology and biotechnology are called three mainstream technologies in 21 century, also the scientific and research hotspot in the whole world. Nano materials refer to materials of $0.1-100 \mathrm{~nm}$. Nano-technology is to apply this kind of materials to conduct the research, design, manufacturing and measurement of products, belonging to cutting-edge technology with high contents of science and education. With current technological level, the machining precision of some machines can reach the level of nano, whereas, this level is just that the appearance of materials reaches the level of nano. Different from traditional machining, nano machining requires the self-structure of materials to reach the level of nano. Current application field of nano-technology is new nano materials and new nano functional appliance, with top-down processing or bottom-up processing. There is certain difficulties in realizing the industrialization of nano technician in current conditions, and bright overall prospect at the same time.

\section{The Optimizing Application of Modern Machinery Manufacturing and Precision Machining}

\subsection{To control the overall situation and realize the integration of technologies}

The application of modern machinery manufacturing and precision machining in the procedures 
of manufacturing technician, firstly, shall include design development, manufacturing, quality inspection, production management, precision machining in initial stage of mechanical equipment, and specific usage, maintenance and troubleshooting in operation of machines. There is a certain difference in technologies in different production links, and necessary relation at the same time. Once there is problem in one link, the normal operation of other links will be impacted, further actual production schedule of enterprises and even economic benefits if it is serious. Therefore, enterprises shall combine with their own actual situations and technological level, formulate reasonable production goal and design scheme, grasp machinery technology and precision machining technology on the whole and realize the integration of various different technologies in manufacturing [8].

\subsection{To strictly monitor the quality of materials and improve comprehensive benefits of enterprises}

At present, the nation strongly advocates environmental protection, in the transformation of economic development mode, enterprise shall strictly control the quality of materials and improve social benefits and economic benefits of hi-tech technology application according to basic principles of the coordinated development of resources in actual production. In the application, it is necessary to comprehensively consider factors in various aspects, reduce environmental pollution and the waste of natural resources as much as possible when selecting materials, to select recyclable materials with high cost performance for production, and use equipment to meet the requirements of less discharge capacity and low energy consumption.

\subsection{To strengthen the training of technicians}

The competition of enterprises is the competition of talents. There is large difference between traditional technology and modern machinery manufacturing and precision machining. In order to give full play to new technology, enterprises shall regularly organize the training of operating staff working at the production line, employ professional experts and technician with a certain experience to teach the use of equipment and knowledge related to operation, cooperate with actual operation practices, and enhance comprehensive capacity of technician to accurately apply new technology for production and serve enterprises. It is also necessary to periodically organize various kinds of competitions of operating skills, give awards to excellent staff, increase their enthusiasm of learning, and promote work efficiency to give play to new technology.

\subsection{To update the concept of operation management}

In the face of fierce market competition, enterprises are required to gradually adjust their concept of operation management, adopt modern management model, apply new technologies to improve the quality of product and production benefits, determine position responsibilities of all people, establish performance evaluation system suitable for enterprises, conduct evaluation on all positions, give awards to personnel with effective work performance and strong application capacity of new technologies and enhance the initiative of all staff. It is also necessary to regularly hold concluding meetings, fully understand problems in practical application of modern machinery manufacturing and precision machining, formulate solution schemes in time and promote the comprehensive transformation and upgrading of enterprises to achieve greater development in new era.

\section{Conclusion}

Along with the development of times and deepening of market economic system, machinery manufacturing industry faces fierce market competition. Therefore, enterprises shall actively conduct technological innovations, vigorously promote modern machinery manufacturing and precision machining, improve the quality of products, uphold the concept of energy conservation and environmental protection, reduce the waste of resources, reduce costs, enhance work efficiency, strengthen the training of technician to give full play to new technologies, and incessantly promote their own comprehensive competitiveness to obtain greater development in new era. 


\section{References}

[1] Liu Xuqin, Wang Dongming, Zhao Xiaoying. Analysis on Common Machinery Manufacturing Technology and Precision Machining Technology [J]. Management \& Technology of SME, 2015(05):241-242.

[2] Wang Guilin. Modern Machinery Manufacturing Technology and Precision Machining Technology [J]. Modern Manufacturing Technology and Equipment, 2016(09):111-112.

[3] Li Guangpei, Lu Yonglong, Li Long. Research on Modern Machinery Manufacturing Technology and Precision Machining Technology [J]. Shandong Industrial Technology, 2016(04):4.

[4] Song Guangjun. On Modern Machinery Manufacturing Technology and Precision Machining Technology [A]. Scientific and research result of national special fund for teachers' scientific research (2) [C]. 2015:2.

[5] Cheng Jianwen. On Machinery Manufacturing Technology and Precision Machining Technology [J]. Management \& Technology of SME, 2017(12):149-150.

[6] Zhou Dong, Zhang Haifei. On Machinery Manufacturing Technology and Precision Machining Technology [J]. Heilongjiang Science, 2017, 8(06):116-117.

[7] Zhao Boxin, Zhou Jian, Zhang Chi. On Machinery Manufacturing Technology and Precision Machining Technology [J]. Telecom World, 2015(06):176.

[8] Chen Peng. Modern Machining Technology and Precision Machining Technology [J]. Modern Manufacturing Technology and Equipment, 2016(12):135-137. 